\title{
Functional Limit Theorems for Toeplitz Quadratic Functionals of Continuous time Gaussian Stationary Processes
}

\author{
Shuyang Bai, Mamikon S. Ginovyan, Murad S. Taqqu \\ Boston University
}

April 24, 2015

\begin{abstract}
The paper establishes weak convergence in $C[0,1]$ of normalized stochastic processes, generated by Toeplitz type quadratic functionals of a continuous time Gaussian stationary process, exhibiting longrange dependence. Both central and non-central functional limit theorems are obtained.
\end{abstract}

Key words. Stationary Gaussian process - Toeplitz-type quadratic functional - Brownian motion - Noncentral limit theorem - Long memory - Wiener-Itô integral.

\section{Introduction}

Let $\{X(t), t \in \mathbb{R}\}$ be a centered real-valued stationary Gaussian process with spectral density $f(x)$ and covariance function $r(t)$, that is, $r(t)=\widehat{f}(t)=\int_{\mathbb{R}} e^{i x t} f(x) d x, t \in \mathbb{R}$. We are interested in describing the limit (as $T \rightarrow \infty$ ) of the following process, generated by Toeplitz type quadratic functionals of the process $X(t)$ :

$$
Q_{T}(t)=\int_{0}^{T t} \int_{0}^{T t} \widehat{g}(u-v) X(u) X(v) d u d v, \quad t \in[0,1]
$$

where

$$
\widehat{g}(t)=\int_{\mathbb{R}} e^{i x t} g(x) d x, \quad t \in \mathbb{R},
$$

is the Fourier transform of some integrable even function $g(x), x \in \mathbb{R}$. We will refer to $g(x)$ and to its Fourier transform $\widehat{g}(t)$ as a generating function and generating kernel for the process $Q_{T}(t)$, respectively.

The limit of the process (1.1) is completely determined by the spectral density $f(x)$ (or covariance function $r(t)$ ) and the generating function $g(x)$ (or generating kernel $\widehat{g}(t)$ ), and depending on their properties, the limit can be either Gaussian (that is, $Q_{T}(t)$ with an appropriate normalization obeys a central limit theorem), or non-Gaussian. The following two questions arise naturally:

(a) Under what conditions on $f(x)$ (resp. $r(t))$ and $g(x)$ (resp. $\widehat{g}(t)$ ) will the limit be Gaussian?

(b) Describe the limit process, if it is non-Gaussian.

Similar questions were considered by Fox and Taqqu [6], Ginovyan and Sahakyan [9], and Terrin and Taqqu [25] in the discrete time case.

Here we work in continuous time, and establish weak convergence in $C[0,1]$ of the process (1.1). The limit processes can be Gaussian or non-Gaussian. The limit non-Gaussian process is identical to that of in the discrete time case, obtained in [25].

But first some brief history. The question (a) goes back to the classical monograph by Grenander and Szegö [16], where the problem was considered for discrete time processes, as an application of the authors' 
theory of the asymptotic behavior of the trace of products of truncated Toeplitz matrices (see [16], p. 217-219). Later the question (a) was studied by Ibragimov [17] and Rosenblatt [23], in connection to the statistical estimation of the spectral function $F(x)$ and covariance function $r(t)$, respectively. Since 1986, there has been a renewed interest in both questions (a) and (b), related to the statistical inferences for long memory processes (see, e.g., Avram [1], Fox and Taqqu [6], Ginovyan and Sahakyan [9], Ginovyan et al. [11], Giraitis et al. [12], Giraitis and Surgailis [13], Giraitis and Taqqu [15], Terrin and Taqqu [26], Taniguchi and Kakizawa [24], and references therein). In particular, Avram [1], Fox and Taqqu [6], Giraitis and Surgailis [13], Ginovyan and Sahakyan [9] have obtained sufficient conditions for the Toeplitz type quadratic forms $Q_{T}(1)$ to obey the central limit theorem (CLT), when the model $X(t)$ is a discrete time process.

For continuous time processes the question (a) was studied in Ibragimov [17] (in connection to the statistical estimation of the spectral function), Ginovyan [7, 8], Ginovyan and Sahakyan [10] and Ginovyan et al. [11], where sufficient conditions in terms of $f(x)$ and $g(x)$ ensuring central limit theorems for quadratic functionals $Q_{T}(1)$ have been obtained.

The rest of the paper is organized as follows. In Section 2 we state the main results of this paper (Theorems $2.1-2.4$ ). In Section 3 we prove a number of preliminary lemmas that are used in the proofs of the main results. Section 4 contains the proofs of the main results.

Throughout the paper the letters $C$ and $c$ with or without indices will denote positive constants whose values can change from line to line.

\section{The Main Results}

In this section we state our main results. Throughout the paper we assume that $f, g \in L^{1}(\mathbb{R})$, and with no loss of generality, that $g \geq 0$ (see [10], [13]).

We first examine the case of central limit theorems, and consider the following standard normalized version of (1.1):

$$
\widetilde{Q}_{T}(t):=T^{-1 / 2}\left(Q_{T}(t)-\mathbb{E}\left[Q_{T}(t)\right]\right), \quad t \in[0,1] .
$$

Our first result, which is an extension of Theorem 1 of [10], involves the convergence of finite-dimensional distributions of the process $\widetilde{Q}_{T}(t)$ to that of a standard Brownian motion.

Theorem 2.1. Assume that the spectral density $f(x)$ and the generating function $g(x)$ satisfy the following conditions:

$$
f \cdot g \in L^{1}(\mathbb{R}) \cap L^{2}(\mathbb{R})
$$

and

$$
\mathbb{E}\left[\widetilde{Q}_{T}^{2}(1)\right] \rightarrow 16 \pi^{3} \int_{\infty}^{\infty} f^{2}(x) g^{2}(x) d x \text { as } T \rightarrow \infty .
$$

Then we have the following convergence of finite-dimensional distributions

$$
\widetilde{Q}_{T}(t) \stackrel{f . d . d .}{\longrightarrow} \sigma B(t)
$$

where $\widetilde{Q}_{T}(t)$ is as in (2.1), B(t) is a standard Brownian motion, and

$$
\sigma^{2}:=16 \pi^{3} \int_{-\infty}^{\infty} f^{2}(x) g^{2}(x) d x
$$

To extend the convergence of finite-dimensional distributions in Theorem 2.1 to the weak convergence in the space $C[0,1]$, we impose an additional condition on the underlying Gaussian process $X(t)$ and on the generating function $g$. It is convenient to impose this condition in the time domain, that is, on the covariance function $r:=\hat{f}$ and the generating kernel $a:=\hat{g}$. The following condition is an analog of the assumption in Theorem 2.3 of [15]:

$$
r(\cdot) \in L^{p}(\mathbb{R}), \quad a(\cdot) \in L^{q}(\mathbb{R}) \quad \text { for some } \quad p, q \geq 1, \frac{1}{p}+\frac{1}{q} \geq \frac{3}{2} .
$$


Remark 2.1. In fact under (2.2), the condition (2.5) is sufficient for the convergence in (2.3). Indeed, let $\bar{p}=p /(p-1)$ be the Hölder conjugate of $p$ and let $\bar{q}=q /(q-1)$ be the Hölder conjugate of $q$. Since $1 \leq p, q \leq 2$, one has by the Hausdorff-Young inequality and (2.5) that $\|f\|_{\bar{p}} \leq c_{p}\|r\|_{p},\|g\|_{\bar{q}} \leq c_{q}\|a\|_{q}$, and hence

$$
f(\cdot) \in L^{\bar{p}}, \quad g(\cdot) \in L^{\bar{q}}, \quad \frac{1}{\bar{p}}+\frac{1}{\bar{q}}=2-\frac{1}{p}-\frac{1}{q} \leq 1 / 2 .
$$

Then the convergence in (2.3) follows from the proof of Theorem 3 from [10]. Note that a similar assertion in the discrete time case was established in [13].

Remark 2.2. Observe that condition (2.5) is fulfilled if the functions $r(t)$ and $a(t)$ satisfy the following: there exist constants $C>0, \alpha^{*}$ and $\beta^{*}$, such that

$$
|r(t)| \leq C\left(1 \wedge|t|^{\alpha^{*}-1}\right), \quad|a(t)| \leq C\left(1 \wedge|t|^{\beta^{*}-1}\right),
$$

where $0<\alpha^{*}, \beta^{*}<1 / 2$ and $\alpha^{*}+\beta^{*}<1 / 2$. Indeed, to see this, note first that $r(\cdot), a(\cdot) \in L^{\infty}(\mathbb{R})$. Then one can choose $p, q \geq 1$ such that $p\left(\alpha^{*}-1\right)<-1$ and $q\left(\beta^{*}-1\right)<-1$, which entails that $r(\cdot) \in L^{p}(\mathbb{R})$ and $a(\cdot) \in L^{q}(\mathbb{R})$. Since $1 / p+1 / q<2-\alpha^{*}-\beta^{*}$ and $2-\alpha^{*}-\beta^{*}>3 / 2$, one can further choose $p, q$ to satisfy $1 / p+1 / q \geq 3 / 2$.

The next results, two functional central limit theorems, extend Theorems 1 and 5 of [10] to weak convergence in the space $C[0,1]$ of the stochastic process $\widetilde{Q}_{T}(t)$ to a standard Brownian motion.

Theorem 2.2. Let the spectral density $f(x)$ and the generating function $g(x)$ satisfy condition (2.2). Let the covariance function $r(t)$ and the generating kernel $a(t)$ satisfy condition (2.5). Then we have the following weak convergence in $C[0,1]$ :

$$
\widetilde{Q}_{T}(t) \Rightarrow \sigma B(t)
$$

where $\widetilde{Q}_{T}(t)$ is as in (2.1), $\sigma$ is as in (2.4), and B(t) is a standard Brownian motion.

Recall that a function $u(x), x \in \mathbb{R}$, is called slowly varying at 0 if it is non-negative and for any $t>0$

$$
\lim _{x \rightarrow 0} \frac{u(x t)}{u(x)} \rightarrow 1
$$

Let $S V_{0}(\mathbb{R})$ be the class of slowly varying at zero functions $u(x), x \in \mathbb{R}$, satisfying the following conditions: for some $a>0, u(x)$ is bounded on $[-a, a], \lim _{x \rightarrow 0} u(x)=0, u(x)=u(-x)$ and $0<u(x)<u(y)$ for $0<x<y<a$. An example of a function belonging to $S V_{0}(\mathbb{R})$ is $u(x)=|\ln | x||^{-\gamma}$ with $\gamma>0$ and $a=1$.

Theorem 2.3. Assume that the functions $f$ and $g$ are integrable on $\mathbb{R}$ and bounded outside any neighborhood of the origin, and satisfy for some $a>0$

$$
f(x) \leq|x|^{-\alpha} L_{1}(x), \quad|g(x)| \leq|x|^{-\beta} L_{2}(x), \quad x \in[-a, a]
$$

for some $\alpha<1, \beta<1$ with $\alpha+\beta \leq 1 / 2$, where $L_{1}(x)$ and $L_{2}(x)$ are slowly varying at zero functions satisfying

$$
L_{i} \in S V_{0}(\mathbb{R}), \quad x^{-(\alpha+\beta)} L_{i}(x) \in L^{2}[-a, a], \quad i=1,2 .
$$

Let, in addition, the covariance function $r(t)$ and the generating kernel a(t) satisfy condition (2.5). Then we have the following weak convergence in $C[0,1]$ :

$$
\widetilde{Q}_{T}(t) \Rightarrow \sigma B(t)
$$

where $\widetilde{Q}_{T}(t)$ is as in (2.1), $\sigma$ is as in (2.4), and $B(t)$ is a standard Brownian motion.

Remark 2.3. The conditions $\alpha<1$ and $\beta<1$ ensure that the Fourier transforms of $f$ and $g$ are well defined. Observe that when $\alpha>0$ the process $\{X(t), t \in \mathbb{Z}\}$ may exhibit long-range dependence. We also allow here $\alpha+\beta$ to assume the critical value $1 / 2$. 
Remark 2.4. The assumptions $f \cdot g \in L^{1}(\mathbb{R}), f, g \in L^{\infty}(\mathbb{R} \backslash[-a, a])$ and $(2.8)$ imply that $f \cdot g \in L^{2}(\mathbb{R})$, so that $\sigma^{2}$ in (2.4) is finite.

Remark 2.5. One may wonder, why, in Theorem 2.3, we suppose that $L_{1}(x)$ and $L_{2}(x)$ belong to $S V_{0}(\mathbb{R})$ instead of merely being slowly varying at zero. This is done in order to deal with the critical case $\alpha+\beta=1 / 2$. Suppose that we are away from this critical case, namely, $f(x)=|x|^{-\alpha} l_{1}(x)$ and $g(x)=|x|^{-\beta} l_{2}(x)$, where $\alpha+\beta<1 / 2$, and $l_{1}(x)$ and $l_{2}(x)$ are slowly varying at zero functions. Assume also that $f(x)$ and $g(x)$ are integrable and bounded on $(-\infty,-a) \cup(a,+\infty)$ for any $a>0$. We claim that Theorem 2.3 applies. Indeed, choose $\alpha^{\prime}>\alpha, \beta^{\prime}>\beta$ with $\alpha^{\prime}+\beta^{\prime}<1 / 2$. Write $f(x)=|x|^{-\alpha^{\prime}}|x|^{\delta} l_{1}(x)$, where $\delta=\alpha^{\prime}-\alpha>0$. Since $l_{1}(x)$ is slowly varying, when $|x|$ is small enough, for some $\epsilon \in(0, \delta)$ we have $|x|^{\delta} l_{1}(x) \leq|x|^{\delta-\epsilon}$. Then one can bound $|x|^{\delta-\epsilon}$ by $c|\ln | x||^{-1} \in S V_{0}(\mathbb{R})$ for small $|x|<1$. Hence one has when $|x|<1$ is small enough, $f(x) \leq|x|^{-\alpha^{\prime}}\left(\left.c|\ln | x\right|^{-1}\right)$. Similarly, when $|x|<1$ is small enough, one has $g(x) \leq|x|^{-\beta^{\prime}}\left(\left.c|\ln | x\right|^{-1}\right)$. All the assumptions in Theorem 2.3 are now readily checked with $\alpha, \beta$ replaced by $\alpha^{\prime}$ and $\beta^{\prime}$, respectively.

Now we state a non-central limit theorem in the continuous time case. Let the spectral density $f$ and the generating function $g$ satisfy

$$
f(x)=|x|^{-\alpha} L_{1}(x) \quad \text { and } \quad g(x)=|x|^{-\beta} L_{2}(x), \quad x \in \mathbb{R}, \quad \alpha<1, \beta<1,
$$

with slowly varying at zero functions $L_{1}(x)$ and $L_{2}(x)$ such that $\int_{\mathbb{R}}|x|^{-\alpha} L_{1}(x) d x<\infty$ and $\int_{\mathbb{R}}|x|^{-\beta} L_{2}(x) d x<$ $\infty$. We assume in addition that the functions $L_{1}(x)$ and $L_{2}(x)$ satisfy the following condition, called Potter's bound (see [12], formula (2.3.5)): for any $\epsilon>0$ there exists a constant $C>0$ so that if $T$ is large enough, then

$$
\frac{L_{i}(u / T)}{L_{i}(1 / T)} \leq C\left(|u|^{\epsilon}+|u|^{-\epsilon}\right), \quad i=1,2 .
$$

Note that a sufficient condition for $(2.10)$ to hold is that $L_{1}(x)$ and $L_{2}(x)$ are bounded on intervals $[a, \infty)$ for any $a>0$, which is the case for the slowly varying functions in Theorem 2.3.

Now we are interested in the limit process of the following normalized version of the process $Q_{T}(t)$ given by (1.1), with $f$ and $g$ as in (2.9):

$$
Z_{T}(t):=\frac{1}{T^{\alpha+\beta} L_{1}(1 / T) L_{2}(1 / T)}\left(Q_{T}(t)-\mathbb{E}\left[Q_{T}(t)\right]\right) .
$$

Theorem 2.4. Let $f$ and $g$ be as in (2.9) with $\alpha<1, \beta<1$ and slowly varying at zero functions $L_{1}(x)$ and $L_{2}(x)$ satisfying (2.10), and let $Z_{T}(t)$ be as in (2.11). Then for $\alpha+\beta>1 / 2$, we have the following weak convergence in the space $C[0,1]$ :

$$
Z_{T}(t) \Rightarrow Z(t)
$$

where the limit process $Z(t)$ is given by

$$
Z(t)=\int_{\mathbb{R}^{2}}^{\prime \prime} H_{t}\left(x_{1}, x_{2}\right) W\left(d x_{1}\right) W\left(d x_{2}\right),
$$

with

$$
H_{t}\left(x_{1}, x_{2}\right)=\left|x_{1} x_{2}\right|^{-\alpha / 2} \int_{\mathbb{R}}\left[\frac{e^{i t\left(x_{1}+u\right)}-1}{i\left(x_{1}+u\right)}\right] \cdot\left[\frac{e^{i t\left(x_{2}-u\right)}-1}{i\left(x_{2}-u\right)}\right]|u|^{-\beta} d u,
$$

where $W(\cdot)$ is a complex Gaussian random measure with Lebesgue control measure, and the double prime in the integral (2.12) indicates that the integration excludes the diagonals $x_{1}= \pm x_{2}$.

Remark 2.6. Comparing Theorem 2.4 and Theorem 1 of [25], we see that the limit process $Z(t)$ is the same both for continuous and discrete time models.

Remark 2.7. Denoting by $P_{T}$ and $P$ the measures generated in $C[0,1]$ by the processes $Z_{T}(t)$ and $Z(t)$ given by (2.11) and (2.12), respectively, Theorem 2.4 can be restated as follows: under the conditions of Theorem 2.4, the measure $P_{T}$ converges weakly in $C[0,1]$ to the measure $P$ as $T \rightarrow \infty$. A similar assertion can be stated for Theorems 2.2 and 2.3 . 
It is worth noting that although the statement of our Theorem 2.4 is similar to that of Theorem 1 of [25], the proof is different and simpler, and does not use the hard analysis of [25], although some technical results of [25] are stated in lemmas and used in the proofs. Our approach in the CLT case (Theorems $2.1-2.3$ ), uses the method developed in [10], which itself is based on an approximation of the trace of the product of truncated Toeplitz operators. For the non-CLT case (Theorem 2.4), we use the integral representation of the underlying process and properties of Wiener-Itô integrals.

\section{Preliminaries}

In this section we state a number of lemmas which will be used in the proof of the theorems. The following result extends Lemma 9 of [10].

Lemma 3.1. Let $Y(t)$ be a centered stationary Gaussian process with spectral density $f_{Y}(x) \in L^{1}(\mathbb{R}) \cap L^{2}(\mathbb{R})$. Consider the normalized process:

$$
L_{T}(t):=\frac{1}{T^{1 / 2}}\left(\int_{0}^{T t} Y^{2}(u) d u-\mathbb{E}\left[\int_{0}^{T t} Y^{2}(u) d u\right]\right) .
$$

Then we have the following convergence of finite-dimensional distributions:

$$
L_{T}(t) \stackrel{f . d . d .}{\longrightarrow} \sigma_{Y} B(t), \quad \sigma_{Y}^{2}=4 \pi \int_{-\infty}^{\infty} f_{Y}^{2}(x) d x
$$

where $B(t)$ is standard Brownian motion.

Remark 3.1. Observe that the normalized processes $\widetilde{Q}_{T}(t)$ and $L_{T}(t)$, given by (2.1) and (3.1), can be expressed by double Wiener-Itô integrals (see, e.g., the proof of Lemma 3.9 below). In our proofs we will use the following fact about weak convergence of multiple Wiener-Itô integrals: given the convergence of the covariance, the multivariate convergence to a Gaussian vector is implied by the univariate convergence of each component (see [21], Proposition 2).

Proof of Lemma 3.1. For a fixed $t$, the univariate convergence in distribution

$$
L_{T}(t) \stackrel{d}{\rightarrow} N\left(0, t \sigma_{Y}^{2}\right) \quad \text { as } \quad T \rightarrow \infty
$$

follows from Lemma 9 of [10]. To show (3.2), in view of Remark 3.1 and Proposition 2 of [21], it remains to show that the covariance structure of $L_{T}(t)$ converges to that of $\sigma_{Y} B(t)$. Specifically, it suffices to show that for any $0<s<t$

$$
\mathbb{E}\left[\left(L_{T}(t)-L_{T}(s)\right)^{2}\right] \rightarrow \sigma_{Y}^{2} \cdot(t-s) \quad \text { as } \quad T \rightarrow \infty .
$$

Indeed, using the fact that for a Gaussian vector $\left(G_{1}, G_{2}\right)$ we have $\operatorname{Cov}\left(G_{1}^{2}, G_{2}^{2}\right)=2\left[\operatorname{Cov}\left(G_{1}, G_{2}\right)\right]^{2}$, and letting $r_{Y}(u)=\int_{\mathbb{R}} e^{i x u} f_{Y}(x) d x$ be the covariance function of $Y(t)$, we can write

$$
\mathbb{E}\left[\left(L_{T}(t)-L_{T}(s)\right)^{2}\right]=2(t-s) \int_{-T(t-s)}^{T(t-s)}\left(1-\frac{|u|}{T(t-s)}\right) r_{Y}^{2}(u) d u
$$

Since $f_{Y}(x) \in L^{2}(\mathbb{R})$, the Fourier transform $r_{Y}(u) \in L^{2}(\mathbb{R})$ as well. So by the Dominated Convergence Theorem and Parseval-Plancherel's identity, we have as $T \rightarrow \infty$

$$
\mathbb{E}\left[\left(L_{T}(t)-L_{T}(s)\right)^{2}\right] \rightarrow 2(t-s) \int_{-\infty}^{\infty} r_{Y}^{2}(u) d u=4 \pi(t-s) \int_{-\infty}^{\infty} f_{Y}^{2}(x) d x=\sigma_{Y}^{2}(t-s) .
$$


We now discuss some results which allow one to reduce the general quadratic functional in Theorem 2.1 to a special quadratic functional introduced in Lemma 3.1.

By Theorem 16.7.2 from [18], the underlying process $X(t)$ admits a moving average representation:

$$
X(t)=\int_{-\infty}^{\infty} \hat{a}(t-s) B(d s) \quad \text { with } \quad \int_{-\infty}^{\infty}|\hat{a}(t)|^{2} d t<\infty
$$

where $B(t)$ is a standard Brownian motion, and $\hat{a}(t)$ is such that its inverse Fourier transform $a(x)$ satisfies $f(x)=2 \pi|a(x)|^{2}$. Assuming the conditions (2.2) and (2.3), we set

$$
b(x)=(2 \pi)^{1 / 2} a(x)(g(x))^{1 / 2},
$$

and observe that the function $b(x)$ is then in $L^{2}(\mathbb{R})$ due to condition $(2.2)$. Consider the stationary process

$$
Y(t)=\int_{-\infty}^{\infty} \hat{b}(t-s) B(d s)
$$

constructed using the Fourier transform $\hat{b}(t)$ of $b(x)$ and the same Brownian motion $B(t)$ as in (3.5). The process $Y(t)$ has spectral density (see [10], equation (4.7))

$$
f_{Y}(x)=2 \pi f(x) g(x) .
$$

We have the following approximation result which immediately follows from Lemma 10 of [10].

Lemma 3.2. Let $\widetilde{Q}_{T}(t)$ be as in (2.1) and let $L_{T}(t)$ be as in (3.1) with $Y(t)$ constructed as in (3.6). Then under the conditions (2.2) and (2.3), for any $t>0$, we have

$$
\lim _{T \rightarrow \infty} \operatorname{Var}\left[\widetilde{Q}_{T}(t)-L_{T}(t)\right]=0 .
$$

The following lemma is a straightforward adaptation of Lemma 4.2 of [14] for functions defined on $\mathbb{R}$.

Lemma 3.3. If $p_{j} \geq 1, j=1, \ldots, k$, where $k \geq 2$ and $\sum_{j=1}^{k} \frac{1}{p_{j}}=k-1$, then

$$
\int_{\mathbb{R}^{k-1}}\left|f_{1}\left(x_{1}\right) \ldots f_{k-1}\left(x_{k-1}\right) f_{k}\left(x_{1}+\ldots+x_{k-1}\right)\right| d x_{1} \ldots d x_{k} \leq \prod_{j=1}^{k}\left\|f_{j}\right\|_{p_{j}} .
$$

The following lemma will be used to establish tightness in the space $C[0,1]$ in Theorem 2.2.

Lemma 3.4. Let the covariance function $r(t)$ and the generating kernel a(t) satisfy condition (2.5), and let $\widetilde{Q}_{T}(t)$ be as in (2.1). Then for all $0 \leq s \leq t \leq 1$ and $T>0$, there exists a constant $C>0$, such that

$$
\mathbb{E}\left[\left|\widetilde{Q}_{T}(t)-\widetilde{Q}_{T}(s)\right|^{2}\right] \leq C(t-s) .
$$

Proof. For convenience we use the Wick product notation: : $X(u) X(v):=X(u) X(v)-\mathbb{E}[X(u) X(v)]$. So for $0 \leq s \leq t \leq 1$, we can write

$$
\begin{aligned}
\widetilde{Q}_{T}(t)-\widetilde{Q}_{T}(s) & =\frac{1}{\sqrt{T}}\left(\int_{0}^{T t} \int_{0}^{T t} a(u-v): X(u) X(v): d u d v-\int_{0}^{T s} \int_{0}^{T s} a(u-v): X(u) X(v): d u d v\right) \\
& =\frac{1}{\sqrt{T}} \int_{T s}^{T t} \int_{T s}^{T t} a(u-v): X(u) X(v): d u d v+\frac{2}{\sqrt{T}} \int_{0}^{T s} \int_{T s}^{T t} a(u-v): X(u) X(v): d u d v \\
& :=A(s, t, T)+B(s, t, T) .
\end{aligned}
$$


Now we estimate $B(s, t, T)$ (the function $A(s, t, T)$ can be estimated similarly). We have by Theorem 3.9 of [19] that

$$
\begin{aligned}
& \mathbb{E}\left[B^{2}(s, t, T)\right]=\frac{4}{T} \int_{0}^{T s} d u_{1} \int_{T s}^{T t} d v_{1} \int_{0}^{T s} d u_{2} \int_{T s}^{T t} d v_{2} a\left(u_{1}-v_{1}\right) a\left(u_{2}-v_{2}\right) \mathbb{E}\left(: X\left(u_{1}\right) X\left(v_{1}\right):: X\left(u_{2}\right) X\left(v_{2}\right):\right) \\
= & \frac{4}{T} \int_{0}^{T s} d u_{1} \int_{T s}^{T t} d v_{1} \int_{0}^{T s} d u_{2} \int_{T s}^{T t} d v_{2} a\left(u_{1}-v_{1}\right) a\left(u_{2}-v_{2}\right)\left[r\left(u_{1}-u_{2}\right) r\left(v_{1}-v_{2}\right)+r\left(u_{1}-v_{2}\right) r\left(v_{1}-u_{2}\right)\right] \\
: & =B_{1}(s, t, T)+B_{2}(s, t, T) .
\end{aligned}
$$

By the change of variables $x_{1}=u_{1}-v_{1}, x_{2}=v_{2}-u_{2}, x_{3}=u_{2}-u_{1}, x_{4}=v_{2}$, and noting that $r(\cdot)$ and $a(\cdot)$ are even functions, we have

$$
B_{1}(s, t, T) \leq \frac{4}{T} \int_{T s}^{T t} d x_{4} \int_{\mathbb{R}^{3}}\left|a\left(x_{1}\right) a\left(x_{2}\right) r\left(x_{3}\right) r\left(x_{1}+x_{2}+x_{3}\right)\right| d x_{1} d x_{2} d x_{3} .
$$

Since $|r(t)| \leq r(0)$, we have $r(\cdot) \in L^{\infty}(\mathbb{R})$. We also have $r(\cdot) \in L^{p}(\mathbb{R})$ by condition $(2.5)$, where $1 / p+1 / q \geq$ $3 / 2$. The $L^{p}$-interpolation theorem states that if a function is in $L^{p_{1}}$ and $L^{p_{2}}$ with $0<p_{1} \leq p_{2} \leq \infty$, then it is in $L^{p^{\prime}}, p_{1} \leq p^{\prime} \leq p_{2}$. By the $L^{p}$-interpolation theorem, one can choose $p^{\prime} \geq p$ such that $r(\cdot) \in L^{p^{\prime}}(\mathbb{R})$ and

$$
\frac{1}{p^{\prime}}+\frac{1}{p^{\prime}}+\frac{1}{q}+\frac{1}{q}=3, \text { that is, } \frac{1}{p^{\prime}}+\frac{1}{q}=\frac{3}{2}
$$

Then by Lemma 3.3 , one has $B_{1}(s, t, T) \leq 4\|r\|_{p^{\prime}}^{2}\|a\|_{q}^{2}(t-s)$. Similarly, one can establish the bound $B_{2}(s, t, T) \leq C(t-s)$, and hence $B(s, t, T) \leq C(t-s)$. So (3.8) is proved.

The lemmas that follow will be used in the proof of Theorem 2.4.

Lemma 3.5. Define

$$
\Delta_{t}(x)=\int_{0}^{t} e^{i s x} d s=\frac{e^{i t x}-1}{i x}
$$

Then for any $\delta \in(0,1)$, there exists a constant $c>0$ depending only on $\delta$, such that

$$
\left|\Delta_{t}(x)\right| \leq c|t|^{\delta} f_{\delta}(x), \quad t \in[0,1], x \in \mathbb{R}
$$

where

$$
f_{\delta}(x)= \begin{cases}|x|^{\delta-1} & \text { if }|x|>1 \\ 1 & \text { if }|x| \leq 1\end{cases}
$$

Proof. In view of (3.9), we have $\left|\Delta_{t}(x)\right| \leq \int_{0}^{t}\left|e^{i s x}\right| d s=t$. So under the constraint $t \in[0,1]$, we have $\left|\Delta_{t}(x)\right| \leq t \leq t^{\delta}$. On the other hand, from Lemma 2 from [25], with some constant $C>0$, we have $\left|e^{i x}-1\right| \leq C|\bar{x}|^{\delta}, \delta \in(0,1)$. So

$$
\left|\Delta_{t}(x)\right| \leq \frac{\left|e^{i t x}-1\right|}{|x|} \leq C|t x|^{\delta}|x|^{-1}=C t^{\delta}|x|^{\delta-1} .
$$

Combining this with (3.11), we obtain (3.10).

We quote Lemma 1 of [25] in a special case, convenient for our purposes.

Lemma 3.6. Let $\gamma_{i}<1, \gamma_{i}+\gamma_{i+1}>1 / 2$, and let $\delta$ be such that

$$
0 \leq \delta<\frac{\gamma_{i}+\gamma_{i+1}}{2}
$$


where $i=1, \ldots, 4$ (with $\left.\gamma_{5}=\gamma_{1}\right)$. Then

$$
\int_{\mathbb{R}^{4}} f_{\delta}\left(y_{1}-y_{2}\right) f_{\delta}\left(y_{2}-y_{3}\right) f_{\delta}\left(y_{3}-y_{4}\right) f_{\delta}\left(y_{4}-y_{1}\right)\left|y_{1}\right|^{-\gamma_{1}}\left|y_{2}\right|^{-\gamma_{2}}\left|y_{3}\right|^{-\gamma_{3}}\left|y_{4}\right|^{-\gamma_{4}} d \mathbf{y}<\infty,
$$

where $f_{\delta}(\cdot)$ is as in (3.11).

Lemma 3.6 can be used to establish the following result.

Lemma 3.7. The function

$$
H_{t}^{*}\left(x_{1}, x_{2}\right):=\left|x_{1}\right|^{\alpha_{1} / 2}\left|x_{2}\right|^{\alpha_{2} / 2} \int_{\mathbb{R}}\left|\Delta_{t}\left(x_{1}+u\right) \Delta_{t}\left(x_{2}-u\right)\right||u|^{-\beta} d u
$$

is in $L^{2}\left(\mathbb{R}^{2}\right)$ for all $\left(\alpha_{1}, \alpha_{2}, \beta\right)$ in the open region $\left\{\left(\alpha_{1}, \alpha_{2}, \beta\right): \alpha_{1}, \alpha_{2}, \beta<1, \alpha_{i}+\beta>1 / 2, i=1,2\right\}$.

Proof. It suffices focus on the case where $t \in[0,1]$, otherwise a change of variable can reduce it to this case. We have by suitable change of variables and Lemma 3.5 that

$$
\begin{aligned}
\left\|H_{t}^{*}\right\|_{L^{2}\left(\mathbb{R}^{2}\right)}^{2} & =\int_{\mathbb{R}^{4}}\left|\Delta_{t}\left(y_{1}-y_{2}\right) \Delta_{t}\left(y_{2}-y_{3}\right) \Delta_{t}\left(y_{3}-y_{4}\right) \Delta_{t}\left(y_{4}-y_{1}\right)\right|\left|y_{1}\right|^{-\alpha_{1}}\left|y_{2}\right|^{-\beta}\left|y_{3}\right|^{-\alpha_{2}}\left|y_{4}\right|^{-\beta} d \mathbf{y} \\
& \leq C \int_{\mathbb{R}^{4}} f_{\delta}\left(y_{1}-y_{2}\right) f_{\delta}\left(y_{2}-y_{3}\right) f_{\delta}\left(y_{3}-y_{4}\right) f_{\delta}\left(y_{4}-y_{1}\right)\left|y_{1}\right|^{-\alpha_{1}}\left|y_{2}\right|^{-\beta}\left|y_{3}\right|^{-\alpha_{2}}\left|y_{4}\right|^{-\beta} d \mathbf{y} .
\end{aligned}
$$

Then apply Lemma 3.6, noting that $\delta$ can be chosen arbitrarily small.

Lemma 3.8. Define the function

$$
H_{t, T}^{*}\left(x_{1}, x_{2}\right)=A_{1, T}\left(x_{1}, x_{2}\right)\left|x_{1} x_{2}\right|^{-\alpha / 2} \int_{\mathbb{R}}\left|\Delta_{t}\left(x_{1}+u\right) \Delta_{t}\left(x_{2}-u\right)\right||u|^{-\beta} A_{2, T}(u) d u,
$$

where

$$
A_{1, T}\left(x_{1}, x_{2}\right)=\sqrt{\frac{L_{1}\left(x_{1} / T\right)}{L_{1}(1 / T)} \frac{L_{1}\left(x_{2} / T\right)}{L_{1}(1 / T)}}, \quad A_{2, T}(u)=\frac{L_{2}(u / T)}{L_{2}(1 / T)} .
$$

Then for large enough $T$, we have $H_{t, T}^{*}\left(x_{1}, x_{2}\right) \in L^{2}\left(\mathbb{R}^{2}\right)$.

Proof. By (2.10) and (3.14), for any $\epsilon>0$ there exists $C>0$, such that for $T$ large enough,

$$
\left|A_{1, T}\left(x_{1}, x_{2}\right)\right| \leq C\left(\left|x_{1}\right|^{\epsilon}+\left|x_{1}\right|^{-\epsilon}\right)\left(\left|x_{2}\right|^{\epsilon}+\left|x_{2}\right|^{-\epsilon}\right)
$$

and

$$
\left|A_{2, T}(u)\right| \leq C\left(|u|^{\epsilon}+|u|^{-\epsilon}\right)
$$

Hence, with some constant $C>0$,

$$
\left|H_{t, T}^{*}\left(x_{1}, x_{2}\right)\right| \leq C \int_{\mathbb{R}}\left|\Delta_{t}\left(x_{1}+u\right) \Delta_{t}\left(x_{2}-u\right)\right||u|^{-\beta}\left(|u|^{\epsilon}+|u|^{-\epsilon}\right) d u\left|x_{1} x_{2}\right|^{-\alpha / 2}\left(\left|x_{1}\right|^{\epsilon}+\left|x_{1}\right|^{-\epsilon}\right)\left(\left|x_{2}\right|^{\epsilon}+\left|x_{2}\right|^{-\epsilon}\right) .
$$

Because by Lemma 3.7, the function $H_{t}^{*}$ in $(3.12)$ is in $L^{2}\left(\mathbb{R}^{2}\right)$ for all $\left(\alpha_{1}, \alpha_{2}, \beta\right)$ in an open region $\{(\alpha, \beta)$ : $\left.\alpha_{1}, \alpha_{2}, \beta<1, \alpha_{i}+\beta>1 / 2, i=1,2\right\}$. By choosing $\epsilon$ small enough, we infer that the right-hand side of (3.17) is in $L^{2}\left(\mathbb{R}^{2}\right)$, and the result follows. 
Lemma 3.9. Let $Z_{T}(t)$ be as in (2.11), and let

$$
Z_{T}^{\prime}(t):=\int_{\mathbb{R}^{2}}^{\prime \prime} H_{t, T}\left(x_{1}, x_{2}\right) W\left(d x_{1}\right) W\left(d x_{2}\right),
$$

where

$$
H_{t, T}\left(x_{1}, x_{2}\right)=A_{1, T}\left(x_{1}, x_{2}\right)\left|x_{1} x_{2}\right|^{-\alpha / 2}\left[\int_{\mathbb{R}} \Delta_{t}\left(x_{1}+u\right) \Delta_{t}\left(x_{2}-u\right)|u|^{-\beta} A_{2, T}(u) d u\right] .
$$

Then $Z_{T}(t) \stackrel{\text { f.d.d. }}{=} Z_{T}^{\prime}(t)$, that is, the processes $Z_{T}(t)$ and $Z_{T}^{\prime}(t)$ have the same finite-dimensional distributions.

Proof. Using the spectral representation of $X(t)$ (see, e.g., [5], Chapter XI, Section 8): $X(t)=\int_{\mathbb{R}} e^{i t x} \sqrt{f(x)} W(d x)$, where $W(\cdot)$ is a complex Gaussian measure with Lebesgue control measure, and the diagram formula (see, e.g., [20], Chapter 5), we have

$$
X(u) X(v)-\mathbb{E}[X(u) X(v)]=\int_{\mathbb{R}^{2}}^{\prime \prime} e^{i\left(u x_{1}+v x_{2}\right)} \sqrt{f\left(x_{1}\right) f\left(x_{2}\right)} W\left(d x_{1}\right) W\left(d x_{2}\right) .
$$

By a stochastic Fubini Theorem (see [22], Theorem 2.1) and Lemma 3.8, one can change the integration order to get (note that by $(1.2)$ we have $\widehat{g}(t)=\int_{\mathbb{R}} e^{i t x} g(x) d x$ ):

$$
\begin{aligned}
Z_{T}(t) & =\frac{1}{T^{\alpha+\beta} L_{1}(1 / T) L_{2}(1 / T)} \int_{\mathbb{R}^{2}}^{\prime \prime} \sqrt{f\left(x_{1}\right) f\left(x_{2}\right)} \int_{0}^{T t} \int_{0}^{T t} \int_{\mathbb{R}} e^{i(u-v) w} g(w) d w e^{i\left(u x_{1}+v x_{2}\right)} d u d v W\left(d x_{1}\right) W\left(d x_{2}\right) \\
& =\frac{1}{T^{\alpha+\beta} L_{1}(1 / T) L_{2}(1 / T)} \int_{\mathbb{R}^{2}}^{\prime \prime} \sqrt{f\left(x_{1}\right) f\left(x_{2}\right)} \int_{\mathbb{R}} \int_{0}^{T t} e^{i u\left(x_{1}+w\right)} d u \int_{0}^{T t} e^{i v\left(x_{2}-w\right)} d v|w|^{-\beta} L(w) d w W\left(d x_{1}\right) W\left(d x_{2}\right) \\
& =\frac{1}{T^{\alpha+\beta} L_{1}(1 / T) L_{2}(1 / T)} \int_{\mathbb{R}^{2}}^{\prime \prime} \sqrt{f\left(x_{1}\right) f\left(x_{2}\right)} \int_{\mathbb{R}} \Delta_{T t}\left(x_{1}+w\right) \Delta_{T t}\left(x_{2}-w\right)|w|^{-\beta} L_{2}(w) d w W\left(d x_{1}\right) W\left(d x_{2}\right) .
\end{aligned}
$$

Now we use the change of variables $w \rightarrow u / T, x_{1} \rightarrow x_{1} / T, x_{2} \rightarrow x_{2} / T$, where the latter two change of variables are subject to the rule $W(d x / T) \stackrel{d}{=} T^{-1 / 2} W(d x)$ (see, e.g., [4], Proposition 4.2), to obtain

$$
\begin{aligned}
& Z_{T}(t) \stackrel{f . d . d .}{=} \frac{1}{T^{\alpha+\beta} L_{1}(1 / T) L_{2}(1 / T)} \times \\
& \int_{\mathbb{R}^{2}}^{\prime \prime} \sqrt{f\left(x_{1} / T\right) f\left(x_{2} / T\right)} \int_{\mathbb{R}} \Delta_{t}\left(x_{1}+u\right) \Delta_{t}\left(x_{2}-u\right)|w / T|^{-\beta} L_{2}(w / T) T d w T^{-1} W\left(d x_{1}\right) W\left(d x_{2}\right) .
\end{aligned}
$$

Taking into account the equality $f(x / T)=|x / T|^{-\alpha} L_{1}(x / T)$ and equations in (3.14), we see that the right hand side of (3.20) coincides with (3.18). This completes the proof.

The lemmas that follow will be used to establish tightness in the space $C[0,1]$ in Theorem 2.4.

Lemma 3.10. Let $\delta$ be a fixed number within the range $(0,(\alpha+\beta) / 2)$, and let $Z_{T}(t)$ be as in (2.11). Then for all $0 \leq s \leq t \leq 1$ and $T$ large enough, there exists a constant $C>0$, such that

$$
\mathbb{E}\left[\left|Z_{T}(t)-Z_{T}(s)\right|^{2}\right] \leq C(t-s)^{2 \delta} .
$$

The same estimate also holds for the corresponding limiting process $Z(t)$ defined by (2.12), (2.13).

Proof. First, in view of Lemma 3.9, we have $\mathbb{E}\left[\left|Z_{T}(t)-Z_{T}(s)\right|^{2}\right]=\mathbb{E}\left[\left|Z_{T}^{\prime}(t)-Z_{T}^{\prime}(s)\right|^{2}\right]$. Next, using the linearity of the multiple stochastic integral, we can write

$$
Z_{T}^{\prime}(t)-Z_{T}^{\prime}(s)=\int_{\mathbb{R}^{2}}^{\prime \prime} H_{s, t, T}\left(x_{1}, x_{2}\right) W\left(d x_{1}\right) W\left(d x_{2}\right),
$$


where

$$
H_{s, t, T}\left(x_{1}, x_{2}\right)=A_{1, T}\left(x_{1}, x_{2}\right)\left|x_{1} x_{2}\right|^{-\alpha / 2} \int_{\mathbb{R}}\left[\Delta_{t}\left(x_{1}+u\right) \Delta_{t}\left(x_{2}-u\right)-\Delta_{s}\left(x_{1}+u\right) \Delta_{s}\left(x_{2}-u\right)\right]|u|^{-\beta} A_{2, T}(u) d u .
$$

The term in the brackets of the integrand in (3.22) can be rewritten as follows:

$$
\begin{aligned}
& \Delta_{t}\left(x_{1}+u\right) \Delta_{t}\left(x_{2}-u\right)-\Delta_{s}\left(x_{1}+u\right) \Delta_{s}\left(x_{2}-u\right) \\
= & \int_{0}^{t} \int_{0}^{t} e^{i w_{1}\left(x_{1}+u\right)} e^{i w_{2}\left(x_{2}-u\right)} d w_{1} d w_{2}-\int_{0}^{s} \int_{0}^{s} e^{i w_{1}\left(x_{1}+u\right)} e^{i w_{2}\left(x_{2}-u\right)} d w_{1} d w_{2} \\
= & \int_{0}^{s} d w_{1} \int_{s}^{t} d w_{2} \ldots+\int_{s}^{t} d w_{1} \int_{0}^{s} d w_{2} \ldots+\int_{s}^{t} d w_{1} \int_{s}^{t} d w_{2} \ldots \\
= & \Delta_{s}\left(x_{1}+u\right) \Delta_{t-s}\left(x_{2}-u\right)+\Delta_{t-s}\left(x_{1}+u\right) \Delta_{s}\left(x_{2}-u\right)+\Delta_{t-s}\left(x_{1}+u\right) \Delta_{t-s}\left(x_{2}-u\right) .
\end{aligned}
$$

Now we apply Lemma 3.5 to get

$$
\begin{aligned}
\left|\Delta_{t}\left(x_{1}+u\right) \Delta_{t}\left(x_{2}-u\right)-\Delta_{s}\left(x_{1}+u\right) \Delta_{s}\left(x_{2}-u\right)\right| & \leq C\left[s^{\delta}(t-s)^{\delta}+(t-s)^{\delta} s^{\delta}+(t-s)^{2 \delta}\right] f_{\delta}\left(x_{1}+u\right) f_{\delta}\left(x_{2}-u\right) \\
& \leq C(t-s)^{\delta} f_{\delta}\left(x_{1}+u\right) f_{\delta}\left(x_{2}-u\right),
\end{aligned}
$$

where the last inequality follows because $0 \leq s^{\delta} \leq 1$ and $0 \leq(t-s)^{\delta} \leq 1$.

Next, using formula $\left(4.5^{\prime}\right)$ of [20], (3.22) and (3.23), we can write

$$
\begin{aligned}
& \mathbb{E}\left[\left|Z_{T}(t)-Z_{T}(s)\right|^{2}\right]=\left\|H_{s, t, T}\right\|_{L^{2}\left(\mathbb{R}^{2}\right)}^{2} \leq C|t-s|^{2 \delta} \int_{\mathbb{R}^{2}} d x_{1} d x_{2} A_{1, T}\left(x_{1}, x_{2}\right)^{2}\left|x_{1} x_{2}\right|^{-\alpha} \times \\
& \int_{\mathbb{R}^{2}} d u_{1} d u_{2} f_{\delta}\left(x_{1}+u_{1}\right) f_{\delta}\left(x_{2}-u_{1}\right) f_{\delta}\left(-x_{1}+u_{2}\right) f_{\delta}\left(-x_{2}-u_{2}\right)\left|u_{1}\right|^{-\beta}\left|u_{2}\right|^{-\beta} A_{2, T}\left(u_{1}\right) A_{2, T}\left(u_{2}\right) \\
\leq & C|t-s|^{2 \delta} \int_{\mathbb{R}^{4}} d y_{1} d y_{2} d y_{3} d y_{4} A_{1, T}\left(y_{1}, y_{3}\right)^{2} A_{2, T}\left(y_{2}\right) A_{2, T}\left(y_{4}\right) \times \\
& f_{\delta}\left(y_{1}-y_{2}\right) f_{\delta}\left(y_{2}-y_{3}\right) f_{\delta}\left(y_{3}-y_{4}\right) f_{\delta}\left(y_{4}-y_{1}\right)\left|y_{1}\right|^{-\alpha}\left|y_{2}\right|^{-\beta}\left|y_{3}\right|^{-\alpha}\left|y_{4}\right|^{-\beta},
\end{aligned}
$$

where we have applied the change of variables: $y_{1}=x_{1}, y_{2}=-u_{1}, y_{3}=-x_{2}, y_{4}=u_{2}$.

Since by assumption $\alpha<1, \beta<1$ and $\alpha+\beta>1 / 2$, and the exponent $\epsilon$ in (3.15) and (3.16) can be chosen arbitrarily small, for a fixed $\delta$ satisfying $0<\delta<(\alpha+\beta) / 2$, we can apply Lemma 1 of [25] to conclude that the integral

$$
\int_{\mathbb{R}^{4}} d \mathbf{y} A_{1, T}\left(y_{1}, y_{3}\right)^{2} A_{2, T}\left(y_{2}\right) A_{2, T}\left(y_{4}\right) f_{\delta}\left(y_{1}-y_{2}\right) f_{\delta}\left(y_{2}-y_{3}\right) f_{\delta}\left(y_{3}-y_{4}\right) f_{\delta}\left(y_{4}-y_{1}\right)\left|y_{1}\right|^{-\alpha}\left|y_{2}\right|^{-\beta}\left|y_{3}\right|^{-\alpha}\left|y_{4}\right|^{-\beta}
$$

is bounded for sufficiently large $T$, which in view of (3.24) implies (3.21). The proof for $Z_{T}(t)$ is thus complete. The proof for $Z(t)$ is similar and so we omit the details.

\section{Proof of Main Results}

Proof of Theorem 2.1. By Lemma 3.2, for any $0 \leq t_{1}<\ldots<t_{n}$, and constants $c_{1}, \ldots, c_{n}$, we have

$$
\lim _{T \rightarrow \infty} \operatorname{Var}\left[\sum_{j=1}^{n} c_{j}\left(\widetilde{Q}_{T}\left(t_{j}\right)-L_{T}\left(t_{j}\right)\right)\right]=0 .
$$

Therefore the convergence of finite-dimensional distributions of $\widetilde{Q}_{T}(t)$ to that of Brownian motion $\sigma B(t)$ follows from Lemma 3.1 with $f_{Y}(\cdot)$ given in (3.7) and the Cramér-Wold Device. 
Proof of Theorem 2.2. In view of the well-known Prokhorov's Theorem (see, e.g., [3], p. 58), to prove the theorem, we need to show convergence of finite-dimensional distributions and tightness. The former has been established in Theorem 2.1. To prove tightness, observe that by Lemma 3.4 and the hypercontractivity inequality of the multiple Wiener-Itô integrals (see [20], Corollary 5.6), for any $T>0$ and $0 \leq s \leq t \leq 1$, there exists a constant $C>0$ to satisfy

$$
\mathbb{E}\left[\left|\widetilde{Q}_{T}(t)-\widetilde{Q}_{T}(s)\right|^{4}\right] \leq C_{2}\left(\mathbb{E}\left[\left|\widetilde{Q}_{T}(t)-\widetilde{Q}_{T}(s)\right|^{2}\right]\right)^{2} \leq C(t-s)^{2} .
$$

Now the tightness of the family of measures generated by the processes $\left\{\widetilde{Q}_{T}(t): T>0\right\}$ in $C[0,1]$ follows from Lemma 5.1 of [17].

Proof of Theorem 2.3. The convergence of finite-dimensional distributions follows from Theorem 2.1. In fact, the assumptions on $f$ and $g$ in Theorem 2.3 imply the conditions (2.2) and (2.3) in Theorem 2.1 (see the proof of Theorem 5 in [10]). The tightness can be shown similarly as in the proof of Theorem 2.2.

Proof of Theorem 2.4. As in the proof of Theorem 2.2, we need to show convergence of finite-dimensional distributions and tightness. We first prove the convergence of finite-dimensional distributions, that is, $Z_{T}(t) \stackrel{f . d . d .}{\longrightarrow} Z(t)$ as $T \rightarrow \infty$, where $Z_{T}(t)$ and $Z(t)$ are defined by $(2.11)$ and (2.12), respectively.

By Lemma 3.9, the process $Z_{T}(t)$ defined in (2.11) has the same finite-dimensional distributions as the process $Z_{T}^{\prime}(t)$ defined in (3.18). Therefore, taking into account the linearity of multiple Wiener-Itô integral, and applying Crámer-Wold device, to prove $Z_{T}(t) \stackrel{\text { f.d.d. }}{\longrightarrow} Z(t)$, it is enough to show that as $T \rightarrow \infty$,

$$
H_{t, T}\left(x_{1}, x_{2}\right) \rightarrow H_{t}\left(x_{1}, x_{2}\right) \quad \text { in } \quad L^{2}\left(\mathbb{R}^{2}\right),
$$

where $H_{t}\left(x_{1}, x_{2}\right)$ and $H_{t, T}\left(x_{1}, x_{2}\right)$ are as in (2.13) and (3.19), respectively.

First, we show pointwise convergence for a.e. $\left(x_{1}, x_{2}\right) \in \mathbb{R}^{2}$, that is,

$$
\begin{aligned}
H_{t, T}\left(x_{1}, x_{2}\right) & =A_{1, T}\left(x_{1}, x_{2}\right)\left|x_{1} x_{2}\right|^{-\alpha / 2} \int_{\mathbb{R}} \Delta_{t}\left(x_{1}+u\right) \Delta_{t}\left(x_{2}-u\right)|u|^{-\beta} A_{2, T}(u) d u \\
& \rightarrow H_{t}\left(x_{1}, x_{2}\right)=\left|x_{1} x_{2}\right|^{-\alpha / 2} \int_{\mathbb{R}} \Delta_{t}\left(x_{1}+u\right) \Delta_{t}\left(x_{2}-u\right)|u|^{-\beta} d u \quad \text { as } T \rightarrow \infty .
\end{aligned}
$$

Because $L_{1}(x)$ is a slowly varying function, we have $A_{1, T}\left(x_{1}, x_{2}\right) \rightarrow 1$ as $T \rightarrow \infty$, where $A_{1, T}$ is as in (3.14). To show that the integral in (4.3) converges to the integral in (4.4), note first that by (3.14), $A_{2, T}(u) \rightarrow 1$ as $T \rightarrow \infty$ because $L_{2}(x)$ is a slowly varying function. Hence one only needs to bound the integrand properly and apply the Dominated Convergence Theorem. To this end, observe that by (3.16) for $T$ large enough, we have

$$
\begin{aligned}
g_{T}\left(u ; x_{1}, x_{2}\right): & =\left|\Delta_{t}\left(x_{1}+u\right)\left\|\Delta_{t}\left(x_{2}-u\right)\right\| u\right|^{-\beta} A_{2, T}(u) \\
& \leq C\left|\Delta_{t}\left(x_{1}+u\right)\left\|\Delta_{t}\left(x_{2}-u\right)\right\| u\right|^{-\beta}\left(|u|^{\epsilon}+|u|^{-\epsilon}\right):=g_{\epsilon}\left(u ; x_{1}, x_{2}\right) .
\end{aligned}
$$

By choosing $\epsilon$ small enough, using Fubini Theorem and Lemma 3.7, we conclude that $g_{\epsilon}\left(\cdot ; x_{1}, x_{2}\right) \in L^{1}(\mathbb{R})$ for a.e. $\left(x_{1}, x_{2}\right) \in \mathbb{R}^{2}$. Now (4.2) follows from (3.17) and the Dominated Convergence Theorem.

To prove tightness, first observe that by the hypercontractivity inequality of the multiple Wiener-Itô integrals (see [20], Corollary 5.6) and Lemma 3.10, for $T$ large enough and for any $0 \leq s \leq t \leq 1$, there exists a constant $C>0$ to satisfy

$$
\mathbb{E}\left[\left|Z_{T}(t)-Z_{T}(s)\right|^{4}\right] \leq C_{2}\left(\mathbb{E}\left[\left|Z_{T}(t)-Z_{T}(s)\right|^{2}\right]\right)^{2} \leq C|t-s|^{4 \delta},
$$

where $\delta$ is a fixed number within the range $0<4 \delta<2(\alpha+\beta)$. Since by assumption $\alpha+\beta>1 / 2$, we can choose $\delta$ to satisfy $4 \delta>1$. Inequalities similar to (4.7) hold also for the limit process $Z(t)$.

In view of (4.7) and a similar inequality for $Z(t)$, it follows from Kolmogorov's criterion (see, e.g., [2] Theorem 8.1(1)) that the processes $Z_{T}(t)$ and $Z(t)$ admit continuous versions when $T$ is large enough.

Now the tightness of the family of measures generated by the processes $\left\{Z_{T}(t): T>0\right\}$ in $C[0,1]$ follows from Lemma 5.1 of [17]. Theorem 2.4 is proved. 
Acknowledgement The research was partially supported by National Science Foundation Grant \#DMS1309009 at Boston University. We would also like to thank the referee for his comments.

\section{References}

[1] Avram, F.: On bilinear forms in Gaussian random variables and Toeplitz matrices. Probability Theory and Related Fields. 79, 37 - 45, 1988.

[2] Bass, R.F.: Stochastic Processes. Cambridge University Press, 2011.

[3] Billingsley, P.: Convergence of Probability Measures. New York, NY: John Wiley \& Sons, Inc, 1999.

[4] Dobrushin, R.L.: Gaussian and their subordinated self-similar random generalized fields. The Annals of Probability, 7(1):1-28, 1979.

[5] Doob, J.L.: Stochastic Processes, volume 101. New York Wiley, 1953.

[6] Fox, R., Taqqu, M.S.: Central limit theorem for quadratic forms in random variables having long-range dependence. Probability Theory and Related Fields. 74, 213 - 240, 1987.

[7] Ginovian, M.S.: On estimate of the value of the linear functional in a spectral density of stationary Gaussian process. Theory of Probability and Its Applications 33, 777 - 781, 1988.

[8] Ginovian, M.S.: On Toeplitz type quadratic functionals in Gaussian stationary process. Probability Theory and Related Fields. 100, 395 - 406, 1994.

[9] Ginovyan, M.S., Sahakyan, A. A.: On the Central Limit Theorem for Toeplitz quadratic forms of stationary sequences. Theory of Probability and Its Applications. 49, 612 - 628, 2005.

[10] Ginovyan, M.S., Sahakyan, A.A.: Limit theorems for Toeplitz quadratic functionals of continuous-time stationary process. Probability Theory and Related Fields. 138, 551-579, 2007.

[11] Ginovyan, M.S., Sahakyan, A.A. and Taqqu, M.S.: The trace problem for Toeplitz matrices and operators and its impact in probability. Probability Surveys. 11, 393-440, 2014.

[12] Giraitis, L, Koul, H.L. and Surgailis, D.: Large Sample Inference for Long Memory Processes. World Scientific Publishing Company Incorporated, 2012.

[13] Giraitis, L., Surgailis, D.: A central limit theorem for quadratic forms in strongly dependent linear variables and its application to asymptotical normality of Whittle's estimate. Probability Theory and Related Fields 86, 87-104, 1990.

[14] Giraitis, L. and Taqqu, M.S.: Central limit theorems for quadratic forms with time-domain conditions. Annals of Probability. 26(1), 377-398, 1998.

[15] Giraitis, L. and Taqqu, M.S: Functional non-central and central limit theorems for bivariate Appell polynomials. Journal of Theoretical Probability, 14(2), 393-426, 2001.

[16] Grenander, U., Szegö, G.: Toeplitz Forms and Their Applications. University of California Press, 1958.

[17] Ibragimov, I.A.: On estimation of the spectral function of a stationary Gaussian process. Theory of Probability and Its Applications. 8, 391 - 430, 1963.

[18] Ibragimov, I.A., Linnik, Yu. V.: Independent and Stationary Sequences of Random Variables. WoltersNoordhoff Publishing Groningen, The Netherlands, 1971.

[19] Janson, S. Gaussian Hilbert Spaces, volume 129 of Cambridge Tracts in Mathematics. Cambridge University Press, 1997. 
[20] Major P.: Multiple Wiener-Itô Integrals. Lecture Notes in Mathematics. Springer, 1981.

[21] Peccati, G. and Tudor, C.: Gaussian limits for vector-valued multiple stochastic integrals. Séminaire de Probabilités XXXVIII, pages 219-245, 2005.

[22] Pipiras, V. and Taqqu, M.S.: Regularization and integral representations of Hermite processes. Statistics and Probability Letters, 80(23):2014-2023, 2010.

[23] Rosenblatt M.: Asymptotic behavior of eigenvalues of Toeplitz forms. Journal of Applied Mathematics and Mechanics 11 941-950, 1962.

[24] Taniguchi, M. and Kakizawa, Y. Asymptotic Theory of Statistical Inference for Time Series. Academic Press, New York, 2000.

[25] Terrin, N and Taqqu, M.S. A noncentral limit theorem for quadratic forms of Gaussian stationary sequences. Journal of Theoretical Probability, 3(3):449-475, 1990.

[26] Terrin, N. and Taqqu, M.S. Convergence to a Gaussian limit as the normalization exponent tends to 1/2. Statistics and Probability Letters 11 419-427, 1991.

Shuyang Bai bsy9142@bu.edu

Mamikon S.Ginovyan ginovyan@math.bu.edu

Murad S. Taqqu murad@bu.edu

Department of Mathematics and Statistics

111 Cumminton Street

Boston, MA, 02215, US 